FACTA UNIVERSITATIS (NIŠ)

Ser. Math. Inform. Vol. 36, No 2 (2021), 333-347

https://doi.org/10.22190/FUMI200724025P

Original Scientific Paper

\title{
LINEAR DIFFERENTIAL POLYNOMIALS WEIGHTED-SHARING A SET OF ROOTS OF UNITY
}

\author{
Dilip Chandra Pramanik and Jayanta Roy \\ Department of Mathematics, University of North Bengal, \\ Raja Rammohunpur, Darjeeling-734013, West Bengal, India
}

\begin{abstract}
In this paper, we study the uniqueness of linear differential polynomials of meromorphic functions when they share a set of roots of unity. Our results shall generalize recent results.

Key words: Meromorphic function, Sharing set, Linear Differential polynomial, Uniqueness.
\end{abstract}

\section{Introduction and main results}

In this paper, by meromorphic function we shall always mean a meromorphic function in the complex plane. We adopt the standard notations in the Nevanlinna Theory of meromorphic functions as explained in $[5,13,14]$. It will be convenient to let $E$ denote any set of positive real numbers of finite linear measure, not necessarily the same at each occurrence.

For any non-constant meromorphic function $f$, we denote by $S(r, f)$ any quantity satisfying $S(r, f)=\circ(T(r, f))$ as $r \rightarrow \infty, r \notin E$. A meromorphic function $a$ is said to be small with respect to $f$ if $T(r, a)=S(r, f)$. We denote by $S(f)$ the collection of all small functions with respect to $f$. Clearly $\mathbb{C} \cup\{\infty\} \subset S(f)$ and $S(f)$ is a field over the set of complex numbers.

Received July 24, 2020, accepted April 5, 2021

Communicated by Hari Mohan Srivastava

Corresponding Author: D. C. Pramanik, Department of Mathematics, University of North Bengal, Raja Rammohunpur, Darjeeling-734013, West Bengal, India | E-mail: dcpramanik.nbu2012@gmail.com

2010 Mathematics Subject Classification. 30D35

(C) 2021 by University of Niš, Serbia | Creative Commons License: CC BY-NC-ND 
For any two non-constant meromorphic functions $f$ and $g$, and $a \in S(f) \cap S(g)$, we say that $f$ and $g$ share $a \operatorname{IM}(\mathrm{CM})$ provided that $f-a$ and $g-a$ have the same zeros ignoring(counting) multiplicities.

During the last few decades the uniqueness theory of entire or meromorphic functions has developed as an active sub-field of the value distribution theory. The main interest of the uniqueness theory is to determine an entire or meromorphic function uniquely satisfying some necessary conditions.

In 1997 Yang and Hua [6] studied the unicity problem for meromorphic functions and differential monomials of the form $f^{n} f^{(1)}$, when they share only one value. S. S. Bhoosnurmath and R. S. Dyavanal [3] extended the Yang-hua's results to the case of $\left(f^{n}\right)^{(k)}$.

Definition 1.1. Let $f$ be a non-constant meromorphic function. An expression of the form

$$
P[f]=\sum_{k=1}^{u} a_{k} \prod_{j=0}^{p}\left(f^{(j)}\right)^{l_{k j}}
$$

where $a_{k} \in S(f)$ for $k=1,2, \ldots \ldots, u$ and $l_{k j}$ are non-negative integers for $k=$ $1,2, \ldots \ldots, u ; j=0,1,2, \ldots ., p$ and $d=\sum_{j=0}^{p} l_{k j}$, for $k=1,2, \ldots \ldots, u$, is called a homogeneous differential polynomial of degree $d$ generated by $f$.

In 2019, Bhoosnurmath, Chakrabarty and Srivastava [4] proved that for a nonconstant homogeneous differential polynomial $P[f]$, the equation $P[f]=1$ has infinitely many zeros.

To state the result we need the following definition.

Definition 1.2. For a meromorphic function $f$ and a set $S \subseteq \mathbb{C}$, we define $E_{f}(S)=\bigcup_{a \in S}\{z \mid f(z)-a=0\}$, counting multiplicities; $\bar{E}_{f}(S)=\bigcup_{a \in S}\{z \mid f(z)-a=$ $0\}$, ignoring multiplicities. If $E_{f}(S)=E_{g}(S)\left(\bar{E}_{f}(S)=\bar{E}_{g}(S)\right)$, then we say that $f$ and $g$ share S CM (IM). Evidently, if $S$ contains only one element then it coincides with the usual definition of CM (respectively IM) shared values.

Recently in 2018 V. H. An and H. H. Khoai [7] have proved the following uniqueness theorem of meromorphic functions.

Theorem 1.1. Let $f$ and $g$ be two non-constant meromorphic functions. Let $k$, $d, n$ be three positive integers with $n>2 k+\frac{2 k+8}{d}, d \geq 2$ and $S=\left\{a \in \mathbb{C}: a^{d}=1\right\}$. If $\left(f^{n}\right)^{(k)}$ and $\left(g^{n}\right)^{(k)}$ share $S C M$, then one of the following holds:

1. $f(z)=c_{1} e^{c z}, g(z)=c_{2} e^{-c z}$, where $c_{1}, c_{2}$ and $c$ are three non-zero constants such that $(-1)^{k d}\left(c_{1} c_{2}\right)^{n d}(n c)^{2 k d}=1$.

2. $f=t g$ for some $t \in \mathbb{C}$ such that $t^{\text {nd }}=1$.

Question 1.1. Regarding Theorem 1.1, a natural question to asked: Can CM be replace by IM keeping the same conclusion? 
In 2020 Dilip et al. answered the above question positively and proved the following theorem.

Theorem 1.2. [11] Let $f$ and $g$ be two non-constant meromorphic functions. Let $k, d, n$ be three positive integers with $n>2 k+\frac{8 k+14}{d}, d \geq 2$ and $S=\left\{a \in \mathbb{C}: a^{d}=\right.$ 1\}. If $\left(f^{n}\right)^{(k)}$ and $\left(g^{n}\right)^{(k)}$ share $S$ IM, then one of the following holds:

1. $f(z)=c_{1} e^{c z}, g(z)=c_{2} e^{-c z}$, where $c_{1}, c_{2}$ and $c$ are three non-zero constants such that $(-1)^{k d}\left(c_{1} c_{2}\right)^{n d}(n c)^{2 k d}=1$.

2. $f=t g$ for some $t \in \mathbb{C}$ such that $t^{\text {nd }}=1$.

Now we recall the notion of weighted sharing which appeared in the literature in $([8,9])$ as this definition paves the way for future discussions as far as relaxation of sharing is concerned. In the following definition, we shall explain this notion.

Definition 1.3. $[8,9]$. Let $l$ be a non-negative integer or infinity and $a \in S(f)$. We denote by $E_{l}(a, f)$ the set of all zeros of $f-a$, where a zero of multiplicity $m$ is counted $m$ times if $m \leq l$ and $l+1$ times if $m>l$. If $E_{l}(a, f)=E_{l}(a, g)$, we say that $f, g$ share the function $a$ with weight $l$. We write $f$ and $g$ share $(a, l)$ to mean that $f$ and $g$ share the function $a$ with weight $l$. Since $E_{l}(a, f)=E_{l}(a, g)$ implies that $E_{s}(a, f)=E_{s}(a, g)$ for any integer $s(0 \leq s<l)$, if $f, g$ share $(a, l)$, then $f, g$ share $(a, s),(0 \leq s<l)$. Moreover, we note that $f$ and $g$ share the function $a$ IM or CM if and only if $f$ and $g$ share $(a, 0)$ or $(a, \infty)$ respectively.

Definition 1.4. Let $S$ be a set of distinct elements of $\mathbb{C} \cup\{\infty\}$ and $l$ be a nonnegative integer or $\infty$. We denote by $E_{f}(S, l)$ the set $E_{f}(S, l)=\bigcup_{a \in S} E_{l}(a, f)$. We say that $f$ and $g$ share the set $S$ with weight $l$ if $E_{f}(S, l)=E_{g}(S, l)$.

Definition 1.5. Let $f$ be a non-constant meromorphic function. Then we denote by $L(f)$ a differential polynomial of the following form: $L(f)=f^{(k)}$ for $k=1,2,3$ and $L(f)=\sum_{j=1}^{k-3} a_{j} f^{(j)}+f^{(k)}$ for $k \geq 4$, where $a_{1}, a_{2}, \ldots ., a_{k-3}$ are constants.

In 2020 Lahiri et al proved the following theorem which improved and generalized Theorem 1.1.

Theorem 1.3. [10] Let $f$ and $g$ be two non-constant meromorphic functions sharing $(\infty, 0)$ and $k, d, n$ be three positive integers with $n>2 k+\frac{2 k+8}{d}, d \geq 2$. Let $S=\left\{a \in \mathbb{C}: a^{d}=1\right\}$. If $L\left(f^{n}\right)$ and $L\left(g^{n}\right)$ share $(S, 2)$ then one of the following holds:

1. $L\left(f^{n}\right)=h L\left(g^{n}\right)$ for some $h \in \mathbb{C}$ such that $h^{d}=1$.

2. $f(z)=c_{1} e^{c z}, g(z)=c_{2} e^{-c z}$, where $c_{1}, c_{2}$ and $c$ are three non-zero constants such that

$$
\left(c_{1} c_{2}\right)^{n}\left\{A \sum_{j=1}^{k-3} a_{j}(n c)^{j}+(n c)^{k}\right\}+\left\{A \sum_{j=1}^{k-3} a_{j}(-n c)^{j}+(-n c)^{k}\right\}=h
$$

and $h^{d}=1$, and $A=0$ if $k=1,2,3$ and $A=1$ if $k \geq 4$. 
In this paper, we shall prove the following result:

Theorem 1.4. Let $f$ and $g$ be two non-constant meromorphic functions sharing $(\infty, 0)$. Let $k(\geq 1), l(\geq 0), d(\geq 2), n(\geq 1)$ be integers and $S=\left\{a \in \mathbb{C}: a^{d}=1\right\}$. If $L\left(f^{n}\right)$ and $L\left(g^{n}\right)$ share $(S, l)$ with one of the following conditions:

(i) $l \geq 2$ and

$$
n>2 k+\frac{2 k+8}{d}
$$

(ii) $l=1$ and

$$
n>2 k+\frac{3 k+9}{d}
$$

(ii) $l=0$ and

$$
n>2 k+\frac{8 k+14}{d}
$$

then one of the following holds:

1. $L\left(f^{n}\right)=h L\left(g^{n}\right)$, where $h^{d}=1$;

2. $f(z)=c_{1} e^{c z}, g(z)=c_{2} e^{-c z}$, where $c_{1}, c_{2}$ and $c$ are three non-zero constants such that

$$
\left(c_{1} c_{2}\right)^{n}\left\{A \sum_{j=1}^{k-3} a_{j}(n c)^{j}+(n c)^{k}\right\}+\left\{A \sum_{j=1}^{k-3} a_{j}(-n c)^{j}+(-n c)^{k}\right\}=h
$$

and $h^{d}=1$, and $A=0$ if $k=1,2,3$ and $A=1$ if $k \geq 4$.

Corollary 1.1. Let $f$ and $g$ be two non-constant entire functions. Let $k(\geq 1)$, $l(\geq 0), d(\geq 2), n(\geq 1)$ be integers and $S=\left\{a \in \mathbb{C}: a^{d}=1\right\}$. If $L\left(f^{n}\right)$ and $L\left(g^{n}\right)$ share $(S, l)$ with one of the following conditions:

(i) $l \geq 2$ and

$$
n>2 k+\frac{2 k+4}{d}
$$

(ii) $l=1$ and

$$
n>2 k+\frac{5 k+9}{2 d}
$$

(ii) $l=0$ and

$$
n>2 k+\frac{5 k+7}{d},
$$

then one of the following holds:

1. $L\left(f^{n}\right)=h L\left(g^{n}\right)$, where $h^{d}=1$; 
2. $f(z)=c_{1} e^{c z}, g(z)=c_{2} e^{-c z}$, where $c_{1}, c_{2}$ and $c$ are three non-zero constants such that

$$
\left(c_{1} c_{2}\right)^{n}\left\{A \sum_{j=1}^{k-3} a_{j}(n c)^{j}+(n c)^{k}\right\}+\left\{A \sum_{j=1}^{k-3} a_{j}(-n c)^{j}+(-n c)^{k}\right\}=h
$$

and $h^{d}=1$, and $A=0$ if $k=1,2,3$ and $A=1$ if $k \geq 4$.

Corollary 1.2. Let $f$ and $g$ be two non-constant meromorphic functions sharing $(\infty, 0)$. Let $k(\geq 1), l(\geq 0), d(\geq 2), n(\geq 1)$ be integers and $S=\left\{a \in \mathbb{C}: a^{d}=1\right\}$. If $\left(f^{n}\right)^{(k)}$ and $\left(g^{n}\right)^{(k)}$ share $(S, l)$ with one of the following conditions:

(i) $l \geq 2$ and

$$
n>\max \left\{3,2 k+\frac{2 k+8}{d}\right\},
$$

(ii) $l=1$ and

$$
n>\max \left\{3,2 k+\frac{3 k+9}{d}\right\},
$$

(ii) $l=0$ and

$$
n>\max \left\{3,2 k+\frac{8 k+14}{d}\right\},
$$

then one of the following holds:

1. $f=\omega g$, where $\omega^{\text {nd }}=1$;

2. $f(z)=c_{1} e^{c z}, g(z)=c_{2} e^{-c z}$, where $c_{1}, c_{2}$ and $c$ are three non-zero constants such that $(-1)^{k d}\left(c_{1} c_{2}\right)^{n d}(n c)^{2 k d}=1$.

\section{Lemmas}

In this section we present some lemmas which will needed in the sequel. Let $F$ and $G$ be non-constant meromorphic functions and $H$ be another function which is defined as follows:

$$
H=\left(\frac{F^{(2)}}{F^{(1)}}-2 \frac{F^{(1)}}{F-1}\right)-\left(\frac{G^{(2)}}{G^{(1)}}-2 \frac{G^{(1)}}{G-1}\right) .
$$

Lemma 2.1. [12, 14] Let $f$ be a non-constant meromorphic function and let $a_{0}, a_{1}$, $\ldots, a_{n}(\not \equiv 0)$ be small functions with respect to $f$. Then

$$
T\left(r, a_{n} f^{n}+a_{n-1} f^{n-1}+\ldots+a_{0}\right)=n T(r, f)+S(r, f) .
$$

Lemma 2.2. [5] Let $f$ be a non-constant meromorphic function and let $k$ be a positive integer. Then

$$
T(r, L(f)) \leq(k+1) T(r, f)+S(r, f) .
$$


Lemma 2.3. [10] Let $f$ be a non-constant meromorphic function and $k, n$ be positive integers with $n \geq k+2, a \in \mathbb{C} \backslash\{0\}$. Then

$$
\frac{n-k-2}{n+k} T(r, f) \leq \bar{N}\left(r, \frac{1}{L\left(f^{n}\right)-a}\right)+S(r, f) \text {. }
$$

Lemma 2.4. [10] Let $f$ be a non-constant meromorphic function and $k, n$ be positive integers with $n>2 k$. Then

(i) $(n-2 k) T(r, f)+k N(r, f)+N\left(r, \frac{f^{n-k}}{L\left(f^{n}\right)}\right) \leq T\left(r, L\left(f^{n}\right)\right)+S(r, f)$.

(ii) $N\left(r, \frac{f^{n-k}}{L\left(f^{n}\right)}\right) \leq k T(r, f)+k \bar{N}(r, f)+S(r, f)$.

Lemma 2.5. [10] Let $f$ and $g$ be two non-constant meromorphic functions sharing $(\infty, 0)$ and $k, n$ be integers with $n \geq k+1$. If $L\left(f^{n}\right) . L\left(g^{n}\right)=h, h \in \mathbb{C} \backslash\{0\}$. Then $f(z)=c_{1} e^{c z}, g(z)=c_{2} e^{-c z}$, where

$$
\left(c_{1} c_{2}\right)^{n}\left\{A \sum_{j=1}^{k-3} a_{j}(n c)^{j}+(n c)^{k}\right\}+\left\{A \sum_{j=1}^{k-3} a_{j}(-n c)^{j}+(-n c)^{k}\right\}=h
$$

and $h^{d}=1$ and $A=0$ if $k=1,2,3$ and $A=1$ if $k \geq 4$.

Lemma 2.6. [1] If $F$ and $G$ be non-constant meromorphic functions sharing $(1,1)$ then

$2 \bar{N}_{L}\left(r, \frac{1}{F-1}\right)+2 \bar{N}_{L}\left(r, \frac{1}{G-1}\right)+\bar{N}_{E}^{(2}\left(r, \frac{1}{F-1}\right)-\bar{N}_{F>2}\left(r, \frac{1}{G-1}\right) \leq N\left(r, \frac{1}{G-1}\right)-\bar{N}\left(r, \frac{1}{G-1}\right)$ $+S(r, F)+S(r, G)$.

Lemma 2.7. [1] If $F$ and $G$ be non-constant meromorphic functions sharing $(1,1)$ then

$\bar{N}_{F>2}\left(r, \frac{1}{G-1}\right) \leq \frac{1}{2} \bar{N}(r, F)+\frac{1}{2} \bar{N}\left(r, \frac{1}{F}\right)-\frac{1}{2} N_{0}\left(r, \frac{1}{F^{(1)}}\right)$.

Lemma 2.8. [2] If $F$ and $G$ be non-constant meromorphic functions sharing $(1,0)$ then $\bar{N}_{L}\left(r, \frac{1}{F-1}\right) \leq \bar{N}(r, F)+\bar{N}\left(r, \frac{1}{F}\right)+S(r, F)$.

Lemma 2.9. Let $l$ be a non-negative integer or infinity. $F$ and $G$ be non-constant meromorphic functions sharing $(1, l)$ and $H$ as defined in (2.1). If $H \not \equiv 0$, then

(i) If $l \geq 2$, then

$$
T(r, F) \leq 2 \bar{N}(r, F)+2 \bar{N}(r, G)+N_{2}\left(r, \frac{1}{F}\right)+N_{2}\left(r, \frac{1}{G}\right)+S(r, F)+S(r, G) .
$$

(ii) If $l=1$, then

$$
\begin{aligned}
T(r, F) \leq & \frac{5}{2} \bar{N}(r, F)+2 \bar{N}(r, G)+N_{2}\left(r, \frac{1}{F}\right)+N_{2}\left(r, \frac{1}{G}\right) \\
& +\frac{1}{2} \bar{N}\left(r, \frac{1}{F}\right)+S(r, F)+S(r, G) .
\end{aligned}
$$


(iii) If $l=0$, then

$$
\begin{gathered}
T(r, F) \leq 4 \bar{N}(r, F)+3 \bar{N}(r, G)+N_{2}\left(r, \frac{1}{F}\right)+N_{2}\left(r, \frac{1}{G}\right) \\
+2 \bar{N}\left(r, \frac{1}{F}+\bar{N}\left(r, \frac{1}{G}\right)+S(r, F)+S(r, G) .\right.
\end{gathered}
$$

The same inequality holds for $T(r, G)$.

Proof. By second fundamental theorem of Nevanlinna we have

$$
\begin{gathered}
T(r, F)+T(r, G) \leq \bar{N}(r, F)+\bar{N}\left(r, \frac{1}{F}\right)+\bar{N}\left(r, \frac{1}{F-1}\right)+\bar{N}(r, G)+\bar{N}\left(r, \frac{1}{G}\right) \\
+\bar{N}\left(r, \frac{1}{G-1}\right)-N_{0}\left(r, \frac{1}{F^{(1)}}\right)-N_{0}\left(r, \frac{1}{G^{(1)}}\right)+S(r, F)+S(r, G),
\end{gathered}
$$

where $N_{0}\left(r, \frac{1}{F^{(1)}}\right)$ denotes the counting function corresponding to the zeros of $F^{(1)}$ which are not the zeros of $F$ and $F-1$. Similarly defined $N_{0}\left(r, \frac{1}{G^{(1)}}\right)$.

We consider the following cases:

Case 1: $l \geq 1$. Then from (2.1) we have

$$
\begin{gathered}
N_{E}^{1)}\left(r, \frac{1}{F-1}\right) \leq N\left(r, \frac{1}{H}\right) \leq T(r, H)+O(1) \leq N(r, H)+S(r, F)+S(r, G) \\
\leq \bar{N}(r, F)+\bar{N}_{(2}\left(r, \frac{1}{F}\right)+\bar{N}(r, G)+\bar{N}_{(2}\left(r, \frac{1}{G}\right)+\bar{N}_{L}\left(r, \frac{1}{F-1}\right)+\bar{N}_{L}\left(r, \frac{1}{G-1}\right) \\
+N_{0}\left(r, \frac{1}{F^{(1)}}\right)+N_{0}\left(r, \frac{1}{G^{(1)}}\right)+S(r, F)+S(r, G),
\end{gathered}
$$

and so

$$
\begin{aligned}
\bar{N}(r, & \left.\frac{1}{F-1}\right)+\bar{N}\left(r, \frac{1}{G-1}\right)=N_{E}^{1)}\left(r, \frac{1}{F-1}\right)+\bar{N}_{L}\left(r, \frac{1}{F-1}\right)+\bar{N}_{L}\left(r, \frac{1}{G-1}\right) \\
+ & \bar{N}_{E}^{(2}\left(r, \frac{1}{F-1}\right)+\bar{N}\left(r, \frac{1}{G-1}\right) \leq \bar{N}(r, F)+\bar{N}(r, G)+\bar{N}_{(2}\left(r, \frac{1}{F}\right) \\
& +\bar{N}_{(2}\left(r, \frac{1}{G}\right)+2 \bar{N}_{L}\left(r, \frac{1}{F-1}\right)+2 \bar{N}_{L}\left(r, \frac{1}{G-1}\right)+\bar{N}_{E}^{(2}\left(r, \frac{1}{F-1}\right) \\
& +\bar{N}\left(r, \frac{1}{G-1}\right)+N_{0}\left(r, \frac{1}{F^{(1)}}\right)+N_{0}\left(r, \frac{1}{G^{(1)}}\right)+S(r, F)+S(r, G) .
\end{aligned}
$$

Subcase 1.1: $l=1$. Using Lemmas 2.6 and 2.7 we get

$$
\begin{gathered}
2 \bar{N}_{L}\left(r, \frac{1}{F-1}\right)+2 \bar{N}_{L}\left(r, \frac{1}{G-1}\right)+\bar{N}_{E}^{(2}\left(r, \frac{1}{F-1}\right)+\bar{N}\left(r, \frac{1}{G-1}\right) \leq N\left(r, \frac{1}{G-1}\right) \\
+\bar{N}_{F>2}\left(r, \frac{1}{G-1}\right) \leq N\left(r, \frac{1}{G-1}\right)+\frac{1}{2} \bar{N}(r, F)+\frac{1}{2} \bar{N}\left(r, \frac{1}{F}\right)-\frac{1}{2} N_{0}\left(r, \frac{1}{F^{(1)}}\right) \\
+S(r, F)+S(r, G) .
\end{gathered}
$$

Thus from (2.3) and (2.4) we have

$$
\begin{gathered}
\bar{N}\left(r, \frac{1}{F-1}\right)+\bar{N}\left(r, \frac{1}{G-1}\right) \leq \bar{N}(r, F)+\bar{N}_{(2}\left(r, \frac{1}{F}\right)+\bar{N}(r, G)+\bar{N}_{(2}\left(r, \frac{1}{G}\right) \\
+N\left(r, \frac{1}{G-1}\right)+\frac{1}{2} \bar{N}(r, F)+\frac{1}{2} \bar{N}\left(r, \frac{1}{F}\right)+\frac{1}{2} N_{0}\left(r, \frac{1}{F^{(1)}}\right)+N_{0}\left(r, \frac{1}{G^{(1)}}\right) \\
+S(r, F)+S(r, G) .
\end{gathered}
$$


Now we deduce from (2.2) and (2.5) that

$$
\begin{gathered}
\quad T(r, F) \leq 2 \bar{N}(r, F)+2 \bar{N}(r, G)+\bar{N}\left(r, \frac{1}{F}\right)+\bar{N}\left(r, \frac{1}{G}\right)+\bar{N}_{(2}\left(r, \frac{1}{F}\right) \\
+\bar{N}_{(2}\left(r, \frac{1}{G}\right)+\frac{1}{2} \bar{N}(r, F)+\frac{1}{2} \bar{N}\left(r, \frac{1}{F}\right)+S(r, F)+S(r, G) \leq \frac{5}{2} \bar{N}(r, F) \\
+2 \bar{N}(r, G)+N_{2}\left(r, \frac{1}{F}\right)+N_{2}\left(r, \frac{1}{G}\right)+\frac{1}{2} \bar{N}\left(r, \frac{1}{F}\right)+S(r, F)+S(r, G) .
\end{gathered}
$$

Subcase 1.2: $l \geq 2$. For this case we have

$$
\begin{gathered}
2 \bar{N}_{L}\left(r, \frac{1}{F-1}\right)+2 \bar{N}_{L}\left(r, \frac{1}{G-1}\right)+\bar{N}_{E}^{(2}\left(r, \frac{1}{F-1}\right)+\bar{N}\left(r, \frac{1}{G-1}\right) \\
\leq N\left(r, \frac{1}{G-1}\right)+S(r, F)+S(r, G) .
\end{gathered}
$$

From (2.2), (2.3) and (2.6), we get

$$
\begin{gathered}
T(r, F) \leq 2 \bar{N}(r, F)+2 \bar{N}(r, G)+\bar{N}\left(r, \frac{1}{F}\right)+\bar{N}\left(r, \frac{1}{G}\right)+\bar{N}_{(2}\left(r, \frac{1}{F}\right) \\
+\bar{N}_{(2}\left(r, \frac{1}{G}\right)+S(r, F)+S(r, G) \leq 2 \bar{N}(r, F)+2 \bar{N}(r, G)+N_{2}\left(r, \frac{1}{F}\right) \\
+N_{2}\left(r, \frac{1}{G}\right)+S(r, F)+S(r, G) .
\end{gathered}
$$

Case 2: $l=0$. Then we have

$$
\begin{aligned}
& N_{E}^{1)}\left(r, \frac{1}{F-1}\right)=N_{E}^{1)}\left(r, \frac{1}{G-1}\right)+S(r, G), \\
& \bar{N}_{E}^{(2}\left(r, \frac{1}{F-1}\right)=\bar{N}_{E}^{(2}\left(r, \frac{1}{G-1}\right)+S(r, G) .
\end{aligned}
$$

From (2.1) we have

$$
\begin{gathered}
\bar{N}\left(r, \frac{1}{F-1}\right)+\bar{N}\left(r, \frac{1}{G-1}\right)=N_{E}^{1)}\left(r, \frac{1}{F-1}\right)+\bar{N}_{E}^{(2}\left(r, \frac{1}{F-1}\right)+\bar{N}_{L}\left(r, \frac{1}{F-1}\right) \\
+\bar{N}_{L}\left(r, \frac{1}{G-1}\right)+\bar{N}\left(r, \frac{1}{G-1}\right) \leq N_{E}^{1)}\left(r, \frac{1}{F-1}\right)+\bar{N}_{L}\left(r, \frac{1}{F-1}\right)+N\left(r, \frac{1}{G-1}\right) \\
\leq \bar{N}(r, F)+\bar{N}(r, G)+\bar{N}_{(2}\left(r, \frac{1}{F}\right)+\bar{N}_{(2}\left(r, \frac{1}{G}\right)+2 \bar{N}_{L}\left(r, \frac{1}{F-1}\right)+\bar{N}_{L}\left(r, \frac{1}{G-1}\right) \\
\quad+N\left(r, \frac{1}{G-1}\right)+N_{0}\left(r, \frac{1}{F^{(1)}}\right)+N_{0}\left(r, \frac{1}{G^{(1)}}\right)+S(r, F)+S(r, G) .
\end{gathered}
$$

By (2.7) and Lemma 2.8 we get from (2.2)

$$
\begin{aligned}
& T(r, F) \leq 2 \bar{N}(r, F)+2 \bar{N}(r, G)+\bar{N}\left(r, \frac{1}{F}\right)+\bar{N}\left(r, \frac{1}{G}\right)+\bar{N}_{(2}\left(r, \frac{1}{F}\right)+\bar{N}_{(2}\left(r, \frac{1}{G}\right) \\
& +2 \bar{N}(r, F)+2 \bar{N}\left(r, \frac{1}{F}\right)+\bar{N}(r, G)+\bar{N}\left(r, \frac{1}{G}\right)+S(r, F)+S(r, G) \leq 4 \bar{N}(r, F) \\
& +3 \bar{N}(r, G)+N_{2}\left(r, \frac{1}{F}\right)+N_{2}\left(r, \frac{1}{G}\right)+2 \bar{N}\left(r, \frac{1}{F}+\bar{N}\left(r, \frac{1}{G}\right)+S(r, F)+S(r, G) .\right.
\end{aligned}
$$

\section{Proof of Main the Theorem}

Proof of Theorem 1.4: 
Proof. Let

$$
F=\left\{L\left(f^{n}\right)\right\}^{d} \text { and } G=\left\{L\left(g^{n}\right)\right\}^{d} .
$$

Since $n \geq k+3$, from Lemma 2.3 with the value 1 , it implies that $L\left(f^{n}\right)=1$ has infinitely many solutions. So $\bar{E}_{L\left(f^{n}\right)}(S) \neq \phi$. Similarly $\bar{E}_{L\left(g^{n}\right)}(S) \neq \phi$. Also by the hypothesis $F, G$ share $(1, l)$.

By Lemmas 2.1, 2.2 and 2.4, we get

$$
\begin{aligned}
(n-2 k) T(r, f) & \leq T\left(r, L\left(f^{n}\right)\right)+S(r, f) \leq(k+1) T\left(r, f^{n}\right)+S(r, f) \\
& \leq(k+1) n T(r, f)+S(r, f)
\end{aligned}
$$

and

$$
\begin{aligned}
(n-2 k) T(r, g) & \leq T\left(r, L\left(g^{n}\right)\right)+S(r, g) \leq(k+1) T\left(r, g^{n}\right)+S(r, g) \\
& \leq(k+1) n T(r, g)+S(r, g) .
\end{aligned}
$$

Also we have

$$
S(r, F)=S\left(r, L\left(f^{n}\right)\right)=S(r, f)
$$

and

$$
S(r, G)=S\left(r, L\left(g^{n}\right)\right)=S(r, g) .
$$

Now, if $a$ is a zero of $L\left(f^{n}\right)$, then $F(a)=0$ with multiplicity $\geq 2$. By (ii) of Lemma 2.4 we get

$$
\begin{gathered}
N_{2}\left(r, \frac{1}{F}\right)=2 \bar{N}\left(r, \frac{1}{L\left(f^{n}\right)}\right) \leq 2 \bar{N}\left(r, \frac{1}{f^{n-k}}\right)+2 N\left(r, \frac{f^{n-k}}{L\left(f^{n}\right)}\right) \\
\leq 2 \bar{N}\left(r, \frac{1}{f}\right)+2 N\left(r, \frac{f^{n-k}}{L\left(f^{n}\right)}\right) \leq 2 T(r, f)+2 N\left(r, \frac{f^{n-k}}{L\left(f^{n}\right)}\right) \\
S(r, f) \leq 2 T(r, f)+2 k T(r, f)+2 k \bar{N}(r, f)+S(r, f) \\
=2(k+1) T(r, f)+2 k \bar{N}(r, f)+S(r, f) . \\
N_{2}\left(r, \frac{1}{G}\right)=2 \bar{N}\left(r, \frac{1}{L\left(g^{n}\right)}\right) \leq 2 T(r, g)+2 N\left(r, \frac{g^{n-k}}{L\left(g^{n}\right)}\right)+S(r, g) \\
\leq 2(k+1) T(r, g)+2 k \bar{N}(r, g)+S(r, g) .
\end{gathered}
$$

Case $1: H \not \equiv 0$. Then by Lemma 2.9 we get following subcases:

Subcase 1.1: If $l \geq 2$, then

$$
\begin{gathered}
T(r, F) \leq N_{2}\left(r, \frac{1}{F}\right)+N_{2}\left(r, \frac{1}{G}\right)+2 \bar{N}(r, F)+2 \bar{N}(r, G) \\
+S(r, F)+S(r, G) .
\end{gathered}
$$

Using (3.1)-(3.4) in (3.5) we get

$$
\begin{gathered}
T\left(r,\left\{L\left(f^{n}\right)\right\}^{d}\right) \leq(2 k+4) \\
T(r, f)+2 k \bar{N}(r, f)+4 T(r, g)+2 N\left(r, \frac{g^{n-k}}{L\left(g^{n}\right)}\right) \\
+S(r, f)+S(r, g) .
\end{gathered}
$$


Similarly,

$$
T\left(r,\left\{L\left(g^{n}\right)\right\}^{d}\right) \leq(2 k+4) T(r, g)+2 k \bar{N}(r, g)+4 T(r, f)+2 N\left(r, \frac{f^{n-k}}{L\left(f^{n}\right)}\right)
$$

Adding (3.6) and (3.7) we obtain

$$
\begin{gathered}
T\left(r,\left\{L\left(f^{n}\right)\right\}^{d}\right)+T\left(r,\left\{L\left(g^{n}\right)\right\}^{d}\right) \leq(2 k+8)\{T(r, f)+T(r, g)\}+2 k\{\bar{N}(r, f) \\
+\bar{N}(r, g)\}+2 N\left(r, \frac{f^{n-k}}{L\left(f^{n}\right)}\right)+2 N\left(r, \frac{g^{n-k}}{L\left(g^{n}\right)}\right)+S(r, f)+S(r, g) .
\end{gathered}
$$

By Lemma 2.4 we get

$$
\begin{gathered}
d\left\{(n-2 k) T(r, f)+k N(r, f)+N\left(r, \frac{f^{n-k}}{L\left(f^{n}\right)}\right)\right\} \\
\leq T\left(r,\left\{L\left(f^{n}\right)\right\}^{d}\right)+S(r, f)
\end{gathered}
$$

and

$$
d\left\{(n-2 k) T(r, g)+k N(r, g)+N\left(r, \frac{g^{n-k}}{L\left(g^{n}\right)}\right)\right\}
$$

$$
\leq T\left(r,\left\{L\left(g^{n}\right)\right\}^{d}\right)+S(r, g) \text {. }
$$

Combining (3.9), (3.10) and using (3.8) we get

$$
\begin{aligned}
d(n- & 2 k)\{T(r, f)+T(r, g)\}+d k\{N(r, f)+N(r, g)\}+d N\left(r, \frac{f^{n-k}}{L\left(f^{n}\right)}\right) \\
+ & d N\left(r, \frac{g^{n-k}}{L\left(g^{n}\right)}\right) \leq(2 k+8)\{T(r, f)+T(r, g)\}+2 N\left(r, \frac{f^{n-k}}{L\left(f^{n}\right)}\right) \\
& +2 k\{\bar{N}(r, f)+\bar{N}(r, g)\}+2 N\left(r, \frac{g^{n-k}}{L\left(g^{n}\right)}\right)+S(r, f)+S(r, g) .
\end{aligned}
$$

Since $d \geq 2$ we have

$$
\begin{aligned}
& d N\left(r, \frac{f^{n-k}}{L\left(f^{n}\right)}\right) \geq 2 N\left(r, \frac{f^{n-k}}{L\left(f^{n}\right)}\right), \\
& d N\left(r, \frac{g^{n-k}}{L\left(g^{n}\right)}\right) \geq 2 N\left(r, \frac{g^{n-k}}{L\left(g^{n}\right)}\right)
\end{aligned}
$$

and

$$
d k\{N(r, f)+N(r, g)\} \geq 2 k\{\bar{N}(r, f)+\bar{N}(r, g)\} .
$$

Using (3.12)-(3.14) we get from (3.11)

$$
d(n-2 k)\{T(r, f)+T(r, g)\} \leq(2 k+8)\{T(r, f)+T(r, g)\}+S(r, f)+S(r, g) .
$$

Therefore $d(n-2 k) \leq 2 k+8 \Rightarrow n \leq 2 k+\frac{2 k+8}{d}$, which contradicts (1.2). 
Subcase $1.2: l=1$, then

$$
\begin{aligned}
T(r, F) \leq & \frac{5}{2} \bar{N}(r, F)+2 \bar{N}(r, G)+N_{2}\left(r, \frac{1}{F}\right)+N_{2}\left(r, \frac{1}{G}\right) \\
& +\frac{1}{2} \bar{N}\left(r, \frac{1}{F}\right)+S(r, F)+S(r, G) .
\end{aligned}
$$

Using (3.1)-(3.4) in (3.15) we get

$$
T\left(r,\left\{L\left(f^{n}\right)\right\}^{d}\right) \leq(3 k+5) T(r, f)+2 k \bar{N}(r, f)+4 T(r, g)+2 N\left(r, \frac{g^{n-k}}{L\left(g^{n}\right)}\right)
$$

$$
+S(r, f)+S(r, g) \text {. }
$$

Similarly,

$$
\begin{gathered}
T\left(r,\left\{L\left(g^{n}\right)\right\}^{d}\right) \leq(3 k+5) \\
T(r, g)+2 k \bar{N}(r, g)+4 T(r, f)+2 N\left(r, \frac{f^{n-k}}{L\left(f^{n}\right)}\right) \\
+S(r, f)+S(r, g) .
\end{gathered}
$$

Adding (3.16) and (3.17) we obtain

$$
\begin{gathered}
T\left(r,\left\{L\left(f^{n}\right)\right\}^{d}\right)+T\left(r,\left\{L\left(g^{n}\right)\right\}^{d}\right) \leq(3 k+9)\{T(r, f)+T(r, g)\}+2 k\{\bar{N}(r, f) \\
(3.18)+\bar{N}(r, g)\}+2 N\left(r, \frac{f^{n-k}}{L\left(f^{n}\right)}\right)+2 N\left(r, \frac{g^{n-k}}{L\left(g^{n}\right)}\right)+S(r, f)+S(r, g) .
\end{gathered}
$$

Combining (3.9), (3.10) and using (3.18) we get

$$
\begin{gathered}
d(n-2 k)\{T(r, f)+T(r, g)\}+d k\{N(r, f)+N(r, g)\}+d N\left(r, \frac{f^{n-k}}{L\left(f^{n}\right)}\right) \\
+d N\left(r, \frac{g^{n-k}}{L\left(g^{n}\right)}\right) \leq(3 k+9)\{T(r, f)+T(r, g)\}+2 N\left(r, \frac{f^{n-k}}{L\left(f^{n}\right)}\right) \\
+2 k\{\bar{N}(r, f)+\bar{N}(r, g)\}+2 N\left(r, \frac{g^{n-k}}{L\left(g^{n}\right)}\right)+S(r, f)+S(r, g) .
\end{gathered}
$$

Using (3.12)-(3.14) we have from (3.19)

$$
d(n-2 k)\{T(r, f)+T(r, g)\} \leq(3 k+9)\{T(r, f)+T(r, g)\}+S(r, f)+S(r, g) .
$$

Therefore $d(n-2 k) \leq 3 k+9 \Rightarrow n \leq 2 k+\frac{3 k+9}{d}$, which contradicts (1.3).

Subcase 1.3: $l=0$, then

$$
\begin{gathered}
T(r, F) \leq 4 \bar{N}(r, F)+3 \bar{N}(r, G)+N_{2}\left(r, \frac{1}{F}\right)+N_{2}\left(r, \frac{1}{G}\right)+2 \bar{N}\left(r, \frac{1}{F}\right) \\
+\bar{N}\left(r, \frac{1}{G}\right)+S(r, F)+S(r, G) .
\end{gathered}
$$

Using (3.1)-(3.4) in (3.20) we get

$$
\begin{gathered}
T\left(r,\left\{L\left(f^{n}\right)\right\}^{d}\right) \leq(6 k+8) T(r, f)+2 k \bar{N}(r, f)+(2 k+6) T(r, g) \\
+2 N\left(r, \frac{g^{n-k}}{L\left(g^{n}\right)}\right)+S(r, f)+S(r, g) .
\end{gathered}
$$


Similarly,

$$
\begin{gathered}
T\left(r,\left\{L\left(g^{n}\right)\right\}^{d}\right) \leq(6 k+8) T(r, g)+2 k \bar{N}(r, g)+(2 k+6) T(r, f) \\
+2 N\left(r, \frac{f^{n-k}}{L\left(f^{n}\right)}\right)+S(r, f)+S(r, g) .
\end{gathered}
$$

Adding (3.21) and (3.22) we obtain

$$
T\left(r,\left\{L\left(f^{n}\right)\right\}^{d}\right)+T\left(r,\left\{L\left(g^{n}\right)\right\}^{d}\right) \leq(8 k+14)\{T(r, f)+T(r, g)\}+2 k\{\bar{N}(r, f)
$$

$$
+\bar{N}(r, g)\}+2 N\left(r, \frac{f^{n-k}}{L\left(f^{n}\right)}\right)+2 N\left(r, \frac{g^{n-k}}{L\left(g^{n}\right)}\right)+S(r, f)+S(r, g) .
$$

By (3.9), (3.10) and (3.23) we get

$$
\begin{aligned}
d(n- & 2 k)\{T(r, f)+T(r, g)\}+d k\{N(r, f)+N(r, g)\}+d N\left(r, \frac{f^{n-k}}{L\left(f^{n}\right)}\right) \\
+ & d N\left(r, \frac{g^{n-k}}{L\left(g^{n}\right)}\right) \leq(8 k+14)\{T(r, f)+T(r, g)\}+2 N\left(r, \frac{f^{n-k}}{L\left(f^{n}\right)}\right) \\
+ & 2 k\{\bar{N}(r, f)+\bar{N}(r, g)\}+2 N\left(r, \frac{g^{n-k}}{L\left(g^{n}\right)}\right)+S(r, f)+S(r, g) .
\end{aligned}
$$

Using (3.12)-(3.14) in (3.24) we obtain

$$
d(n-2 k)\{T(r, f)+T(r, g)\} \leq(8 k+14)\{T(r, f)+T(r, g)\}+S(r, f)+S(r, g) .
$$

Therefore $d(n-2 k) \leq 8 k+14 \Rightarrow n \leq 2 k+\frac{8 k+14}{d}$, which contradicts (1.4).

Case $2: H \equiv 0$. Integrating twice we get

$$
\frac{1}{G-1}=\frac{A}{F-1}+B,
$$

where $A(\neq 0)$ and $B$ are constants.

Thus

$$
G=\frac{(B+1) F+(A-B-1)}{B F+(A-B)}
$$

and

$$
F=\frac{(B-A) G+(A-B-1)}{B G-(B+1)} .
$$

Next we consider the following three subcases : Subcase 2.1: $B \neq 0,-1$. Then from (3.26) we have

$$
\bar{N}\left(r, \frac{1}{G-\frac{B+1}{B}}\right)=\bar{N}(r, F) .
$$

By Nevanlinna second fundamental theorem

$$
\begin{aligned}
T(r, G) & \leq \bar{N}(r, G)+\bar{N}\left(r, \frac{1}{G}\right)+\bar{N}\left(r, \frac{1}{G-\frac{B+1}{B}}\right)+S(r, G) \\
\leq & \bar{N}(r, G)+N_{2}\left(r, \frac{1}{G}\right)+\bar{N}(r, F)+S(r, G)
\end{aligned}
$$




$$
\begin{gathered}
\Rightarrow T\left(r,\left\{L\left(g^{n}\right)\right\}^{d}\right) \leq 2 T(r, g)+2 N\left(r, \frac{g^{n-k}}{L\left(g^{n}\right)}\right)+\bar{N}(r, g) \\
+\bar{N}(r, f)+S(r, f)+S(r, g) .
\end{gathered}
$$

If $A-B-1 \neq 0$, then it follows from (3.25) that

$$
\bar{N}\left(r, \frac{1}{F-\frac{-A+B+1}{B+1}}\right)=\bar{N}\left(r, \frac{1}{G}\right) .
$$

Again by Nevanlinna second fundamental theorem we have

$$
\begin{gathered}
T(r, F) \leq \bar{N}(r, F)+\bar{N}\left(r, \frac{1}{F}\right)+\bar{N}\left(r, \frac{1}{F-\frac{-A+B+1}{B+1}}\right)+S(r, F) \\
\leq \bar{N}(r, F)+N_{2}\left(r, \frac{1}{F}\right)+N_{2}\left(r, \frac{1}{G}\right)+S(r, F)+S(r, G), \\
\Rightarrow T\left(r,\left\{L\left(f^{n}\right)\right\}^{d}\right) \leq \bar{N}(r, f)+2 T(r, f)+2 N\left(r, \frac{f^{n-k}}{L\left(f^{n}\right)}\right) \\
+2 k \bar{N}(r, g)+2(k+1) T(r, g)+S(r, f)+S(r, g) .
\end{gathered}
$$

Combining (3.9), (3.10) and using (3.27), (3.28) we get

$$
\begin{aligned}
d(n- & 2 k)\{T(r, f)+T(r, g)\}+d k\{N(r, f)+N(r, g)\}+d N\left(r, \frac{f^{n-k}}{L\left(f^{n}\right)}\right) \\
+ & d N\left(r, \frac{g^{n-k}}{L\left(g^{n}\right)}\right) \leq 2 T(r, f)+(2 k+5) T(r, g)+2 N\left(r, \frac{f^{n-k}}{L\left(f^{n}\right)}\right) \\
& +2 k\{\bar{N}(r, f)+\bar{N}(r, g)\}+2 N\left(r, \frac{g^{n-k}}{L\left(g^{n}\right)}\right)+S(r, f)+S(r, g) .
\end{aligned}
$$

By using (3.12)-(3.14) in (3.29), we obtain

$$
\begin{gathered}
\left(n-2 k-\frac{2}{d}\right) T(r, f)+\left(n-2 k-\frac{2 k+5}{d}\right) T(r, g) \leq S(r, f)+S(r, g), \\
\Rightarrow\left(n-2 k-\frac{2 k+5}{d}\right)\{T(r, f)+T(r, g)\} \leq S(r, f)+S(r, g),
\end{gathered}
$$

which contradict our assumptions (1.2)-(1.4).

Therefore $A-B-1=0$. Then by $(3.25)$

$$
\bar{N}\left(r, \frac{1}{F+\frac{1}{B}}\right)=\bar{N}(r, G) .
$$

By Nevanlinna second fundamental theorem and Lemma 2.4 we get

$$
\begin{aligned}
T(r, F) & \leq \bar{N}(r, F)+\bar{N}\left(r, \frac{1}{F}\right)+\bar{N}\left(r, \frac{1}{F+\frac{1}{B}}\right)+S(r, F) \\
& \leq \bar{N}(r, F)+N_{2}\left(r, \frac{1}{F}\right)+\bar{N}(r, G)+S(r, F),
\end{aligned}
$$




$$
\begin{aligned}
\Rightarrow T\left(r,\left\{L\left(f^{n}\right)\right\}^{d}\right) & \leq \bar{N}(r, f)+2 T(r, f)+2 N\left(r, \frac{f^{n-k}}{L\left(f^{n}\right)}\right)+\bar{N}(r, g) \\
& +S(r, f)+S(r, g) .
\end{aligned}
$$

Adding (3.9), (3.10) and using (3.27), (3.30) we get

$$
\begin{gathered}
d(n-2 k)\{T(r, f)+T(r, g)\}+d k\{N(r, f)+N(r, g)\} \\
+d N\left(r, \frac{f^{n-k}}{L\left(f^{n}\right)}\right)+d N\left(r, \frac{g^{n-k}}{L\left(g^{n}\right)}\right) \leq 2 T(r, f)+2 T(r, g)+2 N\left(r, \frac{f^{n-k}}{L\left(f^{n}\right)}\right) \\
+2\{\bar{N}(r, f)+\bar{N}(r, g)\}+2 N\left(r, \frac{g^{n-k}}{L\left(g^{n}\right)}\right)+S(r, f)+S(r, g) .
\end{gathered}
$$

By using (3.12)-(3.14) we get from 3.31

$$
\left(n-2 k-\frac{2}{d}\right)\{T(r, f)+T(r, g)\} \leq S(r, f)+S(r, g),
$$

which again violate assumptions (1.2)-(1.4).

Subcase 2.2: $B=-1$. Then

$$
G=\frac{A}{A+1-F}
$$

and

$$
F=\frac{(1+A) G-A}{G}
$$

If $A+1 \neq 0$,

$$
\begin{gathered}
\bar{N}\left(r, \frac{1}{F-(A+1)}\right)=\bar{N}(r, G), \\
\bar{N}\left(r, \frac{1}{G-\frac{A}{A+1}}\right)=\bar{N}\left(r, \frac{1}{F}\right) .
\end{gathered}
$$

By similar argument as Subcase 2.1 we get a contradiction.

Therefore $A+1=0$ then $F G=1 \Rightarrow\left\{L\left(f^{n}\right)\right\}^{d} \cdot\left\{L\left(g^{n}\right)\right\}^{d}=1$. Thus we get $L\left(f^{n}\right) \cdot L\left(g^{n}\right)=h$, where $h^{d}=1$. Then by Lemma 2.5 we get possibility 2 . of the Theorem.

Subcase 2.3: $B=0$. Then (3.25) and (3.26) gives $G=\frac{F+A-1}{A}$ and $F=A G+1-A$ If $A-1 \neq 0, \bar{N}\left(r, \frac{1}{A-1+F}\right)=\bar{N}\left(r, \frac{1}{G}\right)$ and $\bar{N}\left(r, \frac{1}{G-\frac{A-1}{A}}\right)^{A}=\bar{N}\left(r, \frac{1}{F}\right)$. Proceeding similarly as in Subcase 2.1 we get a contradiction.

Therefore, $A-1=0$ then $F \equiv G$ i.e., $L\left(f^{n}\right)=h L\left(g^{n}\right)$ for some $h \in \mathbb{C}$ such that $h^{d}=1$. This completes the proof.

\section{Proof of Corollary 1.2:}


Proof. Putting $L\left(f^{n}\right)=\left(f^{n}\right)^{(k)}$ and $L\left(g^{n}\right)=\left(g^{n}\right)^{(k)}$ we get the following cases from Theorem 1.4.

Case A. $\left(f^{n}\right)^{(k)}=h\left(g^{n}\right)^{(k)}$ where $h^{d}=1$.

By Case I of Theorem 1.2 of [10] we get the possibility 1.

Case B. $\left(f^{n}\right)^{(k)} \cdot\left(g^{n}\right)^{(k)}=h$ where $h^{d}=1$.

For $L\left(f^{n}\right)=\left(f^{n}\right)^{(k)}$ and $L\left(g^{n}\right)=\left(g^{n}\right)^{(k)}$ in Lemma 2.5 we get the possibility 2 .

\section{RE F E R E N C E S}

1. A. BAnerJeE: Meromorphic functions sharing one value. Int. J. Math. Sci. 22 (2005), 3587-3598.

2. A. Banerjee and B. Chakraborty: Further investigations on a questions of Zhang and Lu. Ann. Univ. Paedagog. Crac. Stud. Math. 14 (2015), 105-119.

3. S. S. Bhoosnurmath and R. S. Dyavanal: Uniqueness and value sharing of meromorphic functions. Comput. Math. Appl. 53 (2007), 1191-1205.

4. S. S. Bhoosnurmath, B. Chakraborty and H. M. Srivastava: A Note on the Value Distribution of Differential Polynomials. Commun. Korean Math. Soc. 34(2019), 1145-1155.

5. W. K. Hayman: Meromorphic functions. The Clarendon Press, Oxford 1964.

6. H. X. HUA and C. C. YANG: Uniqueness and value-sharing of meromorphic functions. Ann. Acad. Sci. Fenn. Math. 22 (1997), 395-406.

7. H. H. KHOAI and V. H. AN: On uniqueness for meromorphic functions and their $n$-th derivatives. Annales Univ. Sci. Budapest. Sect. Comp. 47 (2018), 117-126.

8. I. LAHIRI: Weighted value sharing and uniqueness of meromorphic functions. Complex Var. Theory Appl. 46 (2001), 241-253.

9. I. LAHIRI: Weighted sharing and uniqueness of meromorphic functions. Nagoya Math. J. 161 (2001), 193-206.

10. I. LAHIRI and K. SinHA: Linear differential polynomials sharing a set of the roots of unity. Commun. Korean Math. Soc. 35 (2020), No. 3, 773-787.

11. D. C. Pramanik and J. Roy: Uniqueness of Meromorphic Functions Sharing a Set of Roots of Unity. J. Indian. Math. Soc. 87 (3-4) 2020, 206-218.

12. C. C. YANG: On deficiencies of differential polynomials II. Math. Z. 125 (1972), 107-112.

13. L. YANG: Value distributions theory. Springer-Verlag, 1993.

14. H. X. YI and C. C. YANG: Uniqueness theory of meromorphic functions(in Chinese). Science Press, 1995. 